$11-1-2015$

\title{
New Entropy Estimators with Smaller Root Mean Squared Error
}

\author{
Amer Ibrahim Al-Omari \\ Al al-Bayt University, Mafraq, Jordan, alomari_amer@yahoo.com
}

Follow this and additional works at: http://digitalcommons.wayne.edu/jmasm

Part of the Applied Statistics Commons, Social and Behavioral Sciences Commons, and the Statistical Theory Commons

\section{Recommended Citation}

Al-Omari, Amer Ibrahim (2015) "New Entropy Estimators with Smaller Root Mean Squared Error," Journal of Modern Applied Statistical Methods: Vol. 14 : Iss. 2 , Article 10.

DOI: $10.22237 /$ jmasm/1446350940

Available at: http://digitalcommons.wayne.edu/jmasm/vol14/iss2/10

This Regular Article is brought to you for free and open access by the Open Access Journals at DigitalCommons@WayneState. It has been accepted for inclusion in Journal of Modern Applied Statistical Methods by an authorized editor of DigitalCommons@WayneState. 


\section{New Entropy Estimators with Smaller Root Mean Squared Error}

\section{Cover Page Footnote}

The author thanks the editor and the referees for their helpful and valuable comments that substantially improved this paper. 


\title{
New Entropy Estimators with Smaller Root Mean Squared Error
}

\author{
Amer Ibrahim Al-Omari \\ $\mathrm{Al}$ al-Bayt University \\ Mafraq, Jordan
}

New estimators of entropy of continuous random variable are suggested. The proposed estimators are investigated under simple random sampling (SRS), ranked set sampling (RSS), and double ranked set sampling (DRSS) methods. The estimators are compared with Vasicek (1976) and Al-Omari (2014) entropy estimators theoretically and by simulation in terms of the root mean squared error (RMSE) and bias values. The results indicate that the suggested estimators have less RMSE and bias values than their competing estimators introduced by Vasicek (1976) and Al-Omari (2014).

Keywords: Shannon entropy; simple random sampling, ranked set sampling; double ranked set sampling; root mean square error.

\section{Introduction}

The ranked set sampling was first suggested by McIntyre (1952) to estimate a mean of pasture and forage yields. It is a cost efficient sampling procedure alternative to the commonly used simple random sampling scheme. The RSS is useful in situations where the visual ordering of a set of units can be done easily, but the exact measurement of the units is difficult or expensive.

Let the variable of interest $X$ has a probability density function (pdf) $g(x)$ and a cumulative distribution function (cdf) $G(x)$, with mean $\mu$ and variance $\sigma^{2}$. Let $g_{(i: n)}(x)$ and $G_{(i: n)}(x)$ be the pdf and cdf of the $i$ th order statistic, $X_{(i: n)}$, $(1 \leq i \leq n)$ of a random sample of size $n$. The pdf and the cdf of $X_{(i: n)}$, respectively, are given by

$$
g_{(i: n)}(x)=n\left(\begin{array}{c}
n-1 \\
i-1
\end{array}\right) G^{i-1}(x)[1-G(x)]^{n-i} g(x),-\infty<x<\infty
$$

Amer Ibrahim Al-Omari is Faculty of Science, in the Department of Mathematics. Email at:alomari_amer@yahoo.com. 


\section{AMER IBRAHIM AL-OMARI}

and

$$
G_{(i: n)}(x)=\sum_{j=1}^{n}\left(\begin{array}{c}
n \\
j
\end{array}\right) G^{j}(x)[1-G(x)]^{n-j},-\infty<x<\infty
$$

with mean $\mu_{(i: n)}=\int_{-\infty}^{\infty} x g_{(i: n)}(x) d x$ and variance $\sigma_{(i: n)}^{2}=\int_{-\infty}^{\infty}\left(x-\mu_{(i: n)}\right)^{2} g_{(i: n)}(x) d x$.

The ranked set sampling method can be describes as follows:

Step 1. Randomly select $n^{2}$ units from the target population.

Step 2. Allocate the $n^{2}$ selected units randomly into $n$ sets, each of size $n$.

Step 3. Without yet knowing any values for the variable of interest, rank the units within each set with respect to a variable of interest. This may be based on a personal professional judgment or based on a concomitant variable correlated with the variable of interest.

Step 4. The sample units are selected for actual measurement by including the $i$ th smallest ranked unit of the $i$ th sample $(i=1,2, \ldots, n)$.

Step 5. Repeat Steps 1 through 4 for $r$ cycles to obtain a sample of size $n r$ for actual measurement.

It is of interest to note here that even if $n^{2}$ units are selected from the population, but only $n$ of them are measured for comparison with a simple random sampling of the same size $n$.

Let the measured RSS units are denoted by $X_{1(1: n)}, X_{2(2: n)}, \ldots, X_{n(n: n)}$. The RSS estimator of the population mean is defined as $\bar{X}_{R S S}=\frac{1}{n} \sum_{i=1}^{n} X_{i(i: n)}$. Takahasi and Wakimoto (1968) provided the mathematical theory of the RSS and showed that

$$
g(x)=\frac{1}{n} \sum_{i=1}^{n} g_{(i: n)}(x), \mu=\frac{1}{n} \sum_{i=1}^{n} \mu_{(i: n)}, \operatorname{Var}\left(\bar{X}_{R S S}\right)=\frac{\sigma^{2}}{n}-\frac{1}{n^{2}} \sum_{i=1}^{n}\left(\mu_{i(i: n)}-\mu\right)^{2}
$$

Al-Saleh and Al-Kadiri (2000) suggested double ranked set sampling (DRSS) method for estimating the population mean to increase the efficiency of the estimators for fixed sample size. The DRSS method can be described as: 


\section{NEW ENTROPY ESTIMATORS}

Step 1. Randomly choose $n^{2}$ samples of size $n$ each from the target population.

Step 2. Apply the RSS method described above on the $n^{2}$ samples in Step 1. This step yields $n$ samples of size $n$ each.

Step 3. Reapply the RSS method again on the $n$ samples obtained in Step 2 to obtain a sample of size $n$ from the DRSS data. The cycle can be repeated $r$ times if needed to obtain a sample of size $r n$ units.

Let $X$ be a continuous random variable with probability density function $g(x)$ and cumulative distribution function $G(x)$. The entropy $H[g(x)]$ of the random variable is defined by Shannon (1948a, 1948b) as

$$
H[g(x)]=-\int_{-\infty}^{\infty} g(x) \log [g(x)] d x .
$$

The problem of entropy estimation of a continuous random variable is considered by many authors. Vasicek's (1976) suggested an estimator of entropy based on spacing's as

$$
H[g(x)]=\int_{0}^{1} \log \left(\frac{d G^{-1}(p)}{d p}\right) d p,
$$

where the estimation is found by replacing the distribution function $G(x)$ by the empirical distribution function $G_{n}(x)$, and using the difference operator instead of the differential operator. Then the derivative $\frac{d}{d p} G^{-1}(p)$ is estimated by a function of the order statistics.

Let $X_{1}, X_{2}, \ldots, X_{n}$ be a simple random sample of size $n$ from $G(x)$ and $X_{(1)}<X_{(2)}<\ldots<X_{(n)}$ be the order statistics of the sample. Then Vasicek's (1976) estimator of $H[g(x)]$ is defined as

$$
H V_{m n}=\frac{1}{n} \sum_{i=1}^{n} \log \left\{\frac{n}{2 m}\left(X_{(i+m)}-X_{(i-m)}\right)\right\}
$$




\section{AMER IBRAHIM AL-OMARI}

where $m<n / 2$ is a positive integer known as the window size, $X_{(i-\mathrm{m})}=X_{(1)}$ if $i \leq m$, and $X_{(i+\mathrm{m})}=X_{(n)}$ if $i \geq n-m$. He proved that $H V_{m n} \stackrel{P .}{\longrightarrow} H[g(x)]$ as $n \rightarrow \infty, m \rightarrow \infty$, and $\frac{m}{n} \rightarrow 0$.

Van Es (1992) suggested an estimator of entropy based on spacings as

$$
H V E_{m n}=\frac{1}{n-m} \sum_{i=1}^{n-m}\left\{\frac{n+1}{m}\left(X_{(i+m)}-X_{(i)}\right)\right\}+\sum_{k=m}^{n} \frac{1}{k}+\log (m)-\log (n+1)
$$

and proved the consistency and the asymptotic normality of the estimator under some conditions.

Ebrahimi, Pflughoeft, and Soofi (1994) adjusted the weights of Vasicek (1976) estimator to have a smaller weights and proposed an entropy estimator given by

$$
H E_{m n}=\frac{1}{n} \sum_{i=1}^{n} \log \left\{\frac{n}{\theta_{i} m}\left(X_{(i+m)}-X_{(i-m)}\right)\right\}
$$

where

$$
\theta_{i}= \begin{cases}1+\frac{i-1}{m}, & 1 \leq i \leq m, \\ 2, & m+1 \leq i \leq n-m \\ 1+\frac{n-i}{m}, & n-m+1 \leq i \leq n\end{cases}
$$

where $X_{(i-\mathrm{m})}=X_{(1)}$ for $i \leq m$ and $X_{(i+\mathrm{m})}=X_{(n)}$ for $i \geq n-m$. Ebrahimi et al. (1994) showed by simulation that their estimator has a smaller bias and mean squared error than Vasicek (1976) estimator. Also, they proved that

$$
H E_{m n} \stackrel{P .}{\longrightarrow} H[g(x)] \text { as } n \rightarrow \infty, m \rightarrow \infty, m / n \rightarrow 0
$$

Noughabi and Noughabi (2013) suggested a new estimator of entropy of an unknown continuous probability density function as 


\section{NEW ENTROPY ESTIMATORS}

$$
H N N_{m n}=-\frac{1}{n} \sum_{i=1}^{n} \log \left\{s_{i}(n, m)\right\}
$$

where

$$
s_{i}(n, m)= \begin{cases}\hat{g}\left(X_{(i)}\right), & 1 \leq i \leq m, \\ \frac{2 m / n}{X_{(i+m)}-X_{(i-m)}}, & m+1 \leq i \leq n-m, \\ \hat{g}\left(X_{(i)}\right), & n-m+1 \leq i \leq n,\end{cases}
$$

and $\hat{g}\left(X_{i}\right)=\frac{1}{n h} \sum_{j=1}^{n} k\left(\frac{X_{i}-X_{j}}{h}\right)$, where $h$ is bandwidth and $k$ is a kernel function satisfies $\int_{-\infty}^{\infty} k(x) d x=1$. They proved that $H N N_{m n} \stackrel{P}{\longrightarrow} H[g(x)]$ as $n \rightarrow \infty$, $m \rightarrow \infty, m / n \rightarrow 0$. Note that the kernel function in Noughabi and Noughabi (2013) is selected to be the standard normal distribution and the bandwidth $h$ is chosen to be $h=1.06 s n^{-1 / 5}$, where $s$ is the sample standard deviation.

To estimate the entropy $H[g(x)]$ of an unknown continuous probability density function $g(x)$, Noughabi and Arghami (2010) suggested an entropy estimator given by

$$
H N_{m n}=\frac{1}{n} \sum_{i=1}^{n} \log \left\{\frac{n}{c_{i} m}\left(X_{(i+m)}-X_{(i-m)}\right)\right\}
$$

where

$$
c_{i}= \begin{cases}1, & 1 \leq i \leq m, \\ 2, & m+1 \leq i \leq n-m, \\ 1, & n-m+1 \leq i \leq n\end{cases}
$$

and $X_{(i-\mathrm{m})}=X_{(1)}$ if $i \leq m$ and $X_{(i+\mathrm{m})}=X_{(n)}$ for $i \geq n-m$.

Correa (1995) suggested a modified entropy estimator to have smaller mean squared error in the form 


\section{AMER IBRAHIM AL-OMARI}

$$
H C_{m n}=-\frac{1}{n} \sum_{i=1}^{n} \log \left(\frac{\sum_{j=i-m}^{i+m}(j-i)\left(X_{(j)}-\bar{X}_{(i)}\right)}{n \sum_{j=i-m}^{i+m}\left(X_{(j)}-\bar{X}_{(i)}\right)^{2}}\right),
$$

where $\bar{X}_{(i)}=\frac{1}{2 m+1} \sum_{j=i-m}^{i+m} X_{(j)}$.

Al-Omari (2014) suggested three estimators of entropy of an unknown continuous probability density function $g(x)$ using SRS, RSS, and DRSS methods. Based on SRS his first suggested estimator is defined as

$$
\operatorname{AHESRS~}_{m n}=\frac{1}{n} \sum_{i=1}^{n} \log \left\{\frac{n}{\omega_{i} m}\left(X_{(i+m)}-X_{(i-m)}\right)\right\}
$$

where $X_{(i-\mathrm{m})}=X_{(1)}$ for $i \leq m, X_{(i+\mathrm{m})}=X_{(n)}$ for $i \geq n-m$, and

$$
\omega_{i}= \begin{cases}1+\frac{1}{2}, & 1 \leq i \leq m, \\ 2, & m+1 \leq i \leq n-m, \\ 1+\frac{1}{2}, & n-m+1 \leq i \leq n,\end{cases}
$$

The second and third estimators suggested by Al-Omari (2014), based on RSS and DRSS respectively, are given by

$$
\operatorname{AHERSS}_{m n}=\frac{1}{n} \sum_{i=1}^{n} \log \left\{\frac{n}{\omega_{i} m}\left(X_{(i+m)}^{*}-X_{(i-m)}^{*}\right)\right\}
$$

and

$$
\operatorname{AHEDRSS}_{m n}=\frac{1}{n} \sum_{i=1}^{n} \log \left\{\frac{n}{\omega_{i} m}\left(X_{(i+m)}^{* *}-X_{(i-m)}^{* *}\right)\right\}
$$

where $X_{(i-m)}^{*}=X_{(1)}^{*}$ for $i \leq m$ and $X_{(i+m)}^{*}=X_{(n)}^{*}$ for $i \geq n-m$, and 


\section{NEW ENTROPY ESTIMATORS}

$$
X_{(i-m)}^{* *}=X_{(1)}^{* *} \text { for } i \leq m \text { and } X_{(i+m)}^{* *}=X_{(n)}^{* *} \text { for } i \geq n-m \text {. }
$$

For more about entropy estimators, see Choi, Kim, and Song (2004), Park, Park (2003), Goria, Leonenko, Mergel, and Novi Inverardi (2005) and Choi (2008).

The remaining part of this paper is organized as follows. The suggested entropy estimators are given in the section, "Proposed Estimators". Next, a simulation study is conducted to compare the new estimators with their counterparts suggested by Vasicek (1976) and Al-Omari (2014). Finally, some conclusions and suggestions for further works.

\section{The proposed estimators}

The coefficient of the entropy estimators in Ebrahimi et al. (1994), Noughabi and Arghami (2010), and Al-Omari (2014) are adjusted. Let $X_{1}^{(0)}, X_{2}^{(0)}, \ldots, X_{n}^{(0)}$ be a simple random sample of size $n$ from $G(x)$. Based on SRS the first suggested estimator is given by

$$
\operatorname{SHESRS~}_{m n}=\frac{1}{n} \sum_{i=1}^{n} \log \left\{\frac{n}{\varepsilon_{i} m}\left(X_{(i+m)}^{(0)}-X_{(i-m)}^{(0)}\right)\right\}
$$

where

$$
\varepsilon_{i}= \begin{cases}1+\frac{1}{4}, & 1 \leq i \leq m, \\ 2, & m+1 \leq i \leq n-m, \\ 1+\frac{1}{4}, & n-m+1 \leq i \leq n,\end{cases}
$$

$X_{(i-m)}^{(0)}=X_{(1)}^{(0)}$ for $i \leq m$ and $X_{(i+m)}^{(0)}=X_{(n)}^{(0)}$ for $i \geq n-m$. Comparing (3) with (13), we have 


\section{AMER IBRAHIM AL-OMARI}

$$
\begin{aligned}
\operatorname{SHESRS~}_{m n} & =\frac{1}{n} \sum_{i=1}^{n} \log \left\{\frac{n}{\varepsilon_{i} m}\left(X_{(i+m)}^{(0)}-X_{(i-m)}^{(0)}\right)\right\} \\
& =\text { HVSRS }_{m n}+\frac{1}{n} \sum_{i=1}^{n} \log \frac{2}{\varepsilon_{i}} \\
& =\text { HVSRS }_{m n}+\frac{2 m}{n} \log \frac{8}{5}
\end{aligned}
$$

Let $X_{(1: n)}^{(1)}, X_{(2: n)}^{(1)}, \ldots, X_{(n: n)}^{(1)}$ be a RSS of size $n$, Vasicek (1976) entropy estimator using RSS as considered by Mahdizadeh (2012) is given by

$$
\operatorname{HVRSS}_{m n}=\frac{1}{n} \sum_{i=1}^{n} \log \left\{\frac{n}{2 m}\left(X_{(i+m)}^{(1)}-X_{(i-m)}^{(1)}\right)\right\}
$$

Based on the RSS units $X_{(1: n)}^{(1)}, X_{(2: n)}^{(1)}, \ldots, X_{(n: n)}^{(1)}$, the second suggested entropy estimator is

$$
\operatorname{SHERSS}_{m n}=\frac{1}{n} \sum_{i=1}^{n} \log \left\{\frac{n}{\varepsilon_{i} m}\left(X_{(i+m)}^{(1)}-X_{(i-m)}^{(1)}\right)\right\}
$$

where $\varepsilon_{i}$ is defined as in (14), and $X_{(i-m)}^{(1)}=X_{(1)}^{(1)}$ for $i \leq m$ and $X_{(i+m)}^{(1)}=X_{(n)}^{(1)}$ for $i \geq n-m$. Comparing (16) with (17) to have

$$
\begin{aligned}
\text { SHERSS }_{m n} & =\frac{1}{n} \sum_{i=1}^{n} \log \left\{\frac{n}{\varepsilon_{i} m}\left(X_{(i+m)}^{(1)}-X_{(i-m)}^{(1)}\right)\right\} \\
& =\text { HVRSS }_{m n}+\frac{1}{n} \sum_{i=1}^{n} \log \frac{2}{\varepsilon_{i}} \\
& =\text { HVRSS }_{m n}+\frac{2 m}{n} \log \frac{8}{5}
\end{aligned}
$$

Assume that $X_{(1: n)}^{(2)}, X_{(2: n)}^{(2)}, \ldots, X_{(n: n)}^{(2)}$ is a DRSS sample of size $n$. The third suggested entropy estimator has the form 


\section{NEW ENTROPY ESTIMATORS}

$$
\operatorname{SHEDRSS}_{m n}=\frac{1}{n} \sum_{i=1}^{n} \log \left\{\frac{n}{\varepsilon_{i} m}\left(X_{(i+m)}^{(2)}-X_{(i-m)}^{(2)}\right)\right\}
$$

where $\varepsilon_{i}$ is defined as in (14), and $X_{(i-m)}^{(2)}=X_{(1)}^{(2)}$ for $i \leq m$ and $X_{(i+m)}^{(2)}=X_{(n)}^{(2)}$ for $i \geq n-m$. Based on DRSS method Mahdizadeh (2012) showed that Vasicek (1976) estimator will be

$$
\operatorname{SHEDRSS}_{m n}=\frac{1}{n} \sum_{i=1}^{n} \log \left\{\frac{n}{2 m}\left(X_{(i+m)}^{(2)}-X_{(i-m)}^{(2)}\right)\right\}
$$

Comparing (19) with (20) to get

$$
\begin{aligned}
\text { SHEDRSS }_{m n} & =\frac{1}{n} \sum_{i=1}^{n} \log \left\{\frac{n}{\varepsilon_{i} m}\left(X_{(i+m)}^{(2)}-X_{(i-m)}^{(2)}\right)\right\} \\
& =\text { HVDRSS }_{m n}+\frac{1}{n} \sum_{i=1}^{n} \log \frac{2}{\varepsilon_{i}} \\
& =\text { HVDRSS }_{m n}+\frac{2 m}{n} \log \frac{8}{5}
\end{aligned}
$$

Remark 1: The entropy $H\left(f_{n}^{M E}\right)$ of an empirical maximum entropy density $f_{n}^{M E}$ which is related to $H_{V S R} S_{1 n}$ and $\mathrm{SHESRS}_{1 n}$ can be computed following Theil (1980) as:

$$
\begin{aligned}
H\left(f_{n}^{M E}\right) & =\operatorname{HVSRS}_{1 n}+\frac{2-2 \log 2}{n} \\
& =\operatorname{SHESRS}_{1 n}-\frac{2}{n} \log \frac{8}{5}+\frac{2-2 \log 2}{n} \\
& =\text { SHESRS }_{1 n}+\frac{2}{n}\left(1-\log \frac{4}{5}\right)
\end{aligned}
$$

Remark 2: If $n \rightarrow \infty$ in (22), then $H\left(f_{n}^{M E}\right)=S H E S R S_{1 n}$.

In the following two theorems, we compared the suggested estimators with Vasicek (1967) and Al-Omari (2014). 


\section{AMER IBRAHIM AL-OMARI}

Theorem 1: The suggested estimators have the following properties:

a) Let $X_{1}^{(0)}, X_{2}^{(0)}, \ldots, X_{n}^{(0)}$ be SRS of size $n$, then $\operatorname{SHESRS} S_{m n}>H V S R S_{m n}$.

b) Let $X_{(1)}^{(1)}, X_{(2)}^{(1)}, \ldots, X_{(n)}^{(1)}$ be a RSS of size $n$, then HERSS $_{m n}>H V R S S_{m n}$.

c) Let $X_{(1)}^{(2)}, X_{(2)}^{(2)}, \ldots, X_{(n)}^{(2)}$ be a DRSS of size $n$, then $S H E D R S S_{m n}>$ HVDRSS ${ }_{m n}$.

Proof: $\quad$ The proof of (a), (b), (c), is straightforward by using (15), (18), (21), respectively, where $\frac{2 m}{n} \log \frac{8}{5}>0$.

In the following theorem, we compare our suggested entropy estimators with their competitors in Al-Omari (2014).

Theorem 2: Based on the suggested estimators and Al-Omari (2014) entropy respectively, we have

$$
S H E j_{m n}>A H E j_{m n}, j=\mathrm{SRS}, \mathrm{RSS}, \mathrm{DRSS} .
$$

Proof: $\quad$ Compare (9) with (13) based on SRS to obtain

$$
\operatorname{SHESRS}_{m n}-\operatorname{AHESRS}_{m n}=\frac{2 m}{n} \log \frac{6}{5},
$$

and since $\frac{2 m}{n} \log \frac{6}{5}>0$, then the case of SRS holds. Also, compare (11) with (17) based on RSS, and (12) with (19) using DRSS to complete the proof of this theorem.

The following theorem proves the consistency of the suggested estimators $\operatorname{SHESRS}_{m n}, \operatorname{SHERSS}_{m n}$, and SHEDRSS $S_{m n}$.

Theorem 3: Let $\Omega$ be the class of continuous densities with finite entropies and let $X_{1}, X_{2}, \ldots, X_{n}$ be a random sample from $g \in \Omega$. If $n \rightarrow \infty, m \rightarrow \infty, m / n \rightarrow 0$, then $S H E j_{m n},(j=\mathrm{SRS}, \mathrm{RSS}, \mathrm{DRSS})$ converges in probability to $H[g(x)]$.

Proof: $\quad$ Based on the simple random sampling, from (15) we have 


\section{NEW ENTROPY ESTIMATORS}

$$
\operatorname{SHESRS}_{m n}=\operatorname{HVSRS}_{m n}+\frac{2 m}{n} \log \frac{8}{5}
$$

and Vasicek (1976) showed that $\operatorname{HVSRS}_{m n}$ converges in probability to $H[g(x)]$ and since $\frac{2 m}{n} \log \frac{8}{5}$ converges to zero as $n$ goes to infinity, then we proved the case of the SRS. Follow the same approach and use (18) and (21) to prove the theorem for RSS and DRSS estimators, respectively.

\section{Methodology}

\section{Simulation study}

A simulation was conducted to investigate the performance of the suggested entropy estimators with Vasicek (1976) and Al-Omari (2014) entropy estimators using sampling methods considered in this study. The comparison is based on the root mean squared errors (RMSEs) and bias values of the estimators for 10000 samples generated from the uniform, exponential and the standard normal distributions using SRS, RSS and DRSS methods. The selection of the optimal values of the window size of $m$ for a given value $n$ is as yet an open problem in the entropy estimation. Therefore, we used the heuristic formula $m=\sqrt{n}+0.5$ suggested by Wieczorkowski and Grzegorzewski (1999) to select $m$ and to compute the RMSEs of entropy estimators. In this study, we considered the sample and window sizes as given in Table 1.

Table 1. The sample and window sizes considered in this simulation

\begin{tabular}{|c|c|c|c|}
\hline Sample size & $n=10$ & $n=20$ & $n=30$ \\
\hline Window size & $1 \leq m \leq 5$ & $1 \leq m \leq 10$ & $1 \leq m \leq 15$ \\
\hline
\end{tabular}

Also, the performance of the RMSE of the suggested estimators for samples generated from the uniform, exponential and standard normal distributions is evaluated based on the quantity

$$
Q_{N}=\frac{H V j_{m n}-N}{H V j_{m n}} \times 100, N=S H E j_{m n}, A H E j_{m n}, j=S R S, R S S, D R S S .
$$




\section{AMER IBRAHIM AL-OMARI}

The results are summarized in Tables 2-6. Also, we compared the suggested estimators of entropy with their competitors suggested by Al-Omari (2014) and the results presented in Table 7 are taken from Al-Omari (2014).

Based on these results observe the following.

- The suggested entropy estimators using SRS, RSS and DRSS methods are more efficient than their competitors $H V_{m n}$ based on the same method for all cases considered in this study. As an example, from Table 3, with $n=10$ and $m=3$ for the exponential distribution with $H[g(x)]=1$ using RSS method, the RMSE and bias value of SHERSS $_{m n}$ are 0.230412 and -0.052759 compared to 0.401125 and -0.332760 the RMSE and bias of $H V R S S_{m n}$.

- The SHEDRSS ${ }_{m n}$ is superior to the other suggested estimators, $S_{\text {SHERSS }} n$ and SHESRS ${ }_{m n}$ under the uniform, exponential and normal distributions. From Table 1, consider the case of $n=20$ and $m=4$ under the uniform distribution when $H[g(x)]=0$, it can be noted that the RMSE values of SHEDRSS $m$, SHERSS ${ }_{m n}$, and SHESRS $_{m n}$ are $0.052373,0.068747$ and 0.114983 , respectively.

- The nature of the underlying distribution as well as the value of $H[g(x)]$ affect on the efficiency of the estimator using the same method. As an example, the $Q_{\text {SHERSS }_{n n}}$ values with $n=30$ and $m=3$ for the uniform, exponential, and the standard normal distributions are $95.39025,31.76442$ and 32.75544 , respectively. However, the values of $Q_{S H E_{n n}}$ for the uniform distribution with $H[g(x)]=0$ are superior to their counterparts for the exponential and normal distributions.

- Finally, the suggested entropy estimators are found to be more efficient than their competitors in Al-Omari (2014) entropy estimators using SRS, RSS and DRSS schemes for the same window and sample sizes. For illustration, assume that $n=30$ and $m=8$ when the underlying distribution is the standard normal, from Table 4, the RMSE of SHERSS ${ }_{m n}$ is 0.120242 compared to 0.157726 which is the RMSE of AHERSS ${ }_{m n}$ as shown in Table 7. 


\section{NEW ENTROPY ESTIMATORS}

Table 2. The Monte Carlo RMSEs and bias values of $H V_{m n}$ and $S H E_{m n}$ for the uniform distribution with $H[g(x)]=0$.

\begin{tabular}{|c|c|c|c|c|c|c|c|c|c|c|c|}
\hline \multirow[b]{3}{*}{$n$} & \multirow[b]{3}{*}{$m$} & \multicolumn{5}{|c|}{ SRS } & \multicolumn{5}{|c|}{ RSS } \\
\hline & & \multicolumn{2}{|c|}{$H V_{m n}$} & \multicolumn{2}{|c|}{$S H E_{m n}$} & \multirow[t]{2}{*}{$Q_{S H E_{m n}}$} & \multicolumn{2}{|c|}{$H V_{m n}$} & \multicolumn{2}{|c|}{$S H E_{m n}$} & \multirow[t]{2}{*}{$Q_{S H E_{m n}}$} \\
\hline & & Bias & RMSE & Bias & RMSE & & Bias & RMSE & Bias & RMSE & \\
\hline \multirow[t]{5}{*}{10} & 1 & -0.519826 & 0.569537 & -0.430151 & 0.490404 & 13.89427 & -0.396308 & 0.443439 & -0.303703 & 0.361606 & 22.63043 \\
\hline & 2 & -0.415135 & 0.452358 & -0.226627 & 0.290240 & 35.83843 & -0.304078 & 0.329233 & -0.116915 & 0.172961 & 90.35100 \\
\hline & 3 & -0.422613 & 0.453818 & -0.135797 & 0.213148 & 53.03227 & -0.327681 & 0.343991 & -0.045891 & 0.114159 & 201.3262 \\
\hline & 4 & -0.458940 & 0.487054 & -0.080015 & 0.179669 & 63.11107 & -0.371538 & 0.383103 & 0.004574 & 0.093383 & 310.24920 \\
\hline & 5 & -0.502063 & 0.527918 & -0.032713 & 0.167982 & 68.18029 & -0.425903 & 0.436521 & 0.042936 & 0.105150 & 315.14120 \\
\hline \multirow[t]{10}{*}{20} & 1 & -0.393900 & 0.418346 & -0.349192 & 0.376728 & 9.94822 & -0.343340 & 0.365754 & -0.294874 & 0.320679 & 14.05611 \\
\hline & 2 & -0.271880 & 0.290818 & -0.177492 & 0.204940 & 29.52981 & -0.217937 & 0.233026 & -0.125116 & 0.150017 & 55.33306 \\
\hline & 3 & -0.253931 & 0.270200 & -0.112786 & 0.145519 & 46.14397 & -0.205321 & 0.216879 & -0.063859 & 0.093348 & 132.33380 \\
\hline & 4 & -0.260596 & 0.274678 & -0.074069 & 0.114983 & 58.13898 & -0.214042 & 0.222524 & -0.026611 & 0.068747 & 223.68540 \\
\hline & 5 & -0.276800 & 0.288985 & -0.043624 & 0.095299 & 67.02286 & -0.235141 & 0.242179 & 0.000439 & 0.052744 & 359.15930 \\
\hline & 6 & -0.299321 & 0.310256 & -0.017934 & 0.085705 & 72.37604 & -0.258899 & 0.264554 & 0.022973 & 0.059480 & 344.77810 \\
\hline & 7 & -0.322084 & 0.332301 & 0.005663 & 0.082331 & 75.22397 & -0.285310 & 0.290156 & 0.043299 & 0.067712 & 328.51490 \\
\hline & 8 & -0.348254 & 0.357901 & 0.028228 & 0.087902 & 75.43958 & -0.314138 & 0.318471 & 0.061191 & 0.081194 & 292.23460 \\
\hline & 9 & -0.374620 & 0.383864 & 0.048022 & 0.097710 & 74.54567 & -0.343410 & 0.347711 & 0.079914 & 0.096721 & 259.49900 \\
\hline & 10 & -0.402840 & 0.411741 & 0.066866 & 0.108377 & 73.67836 & -0.371780 & 0.375737 & 0.097578 & 0.112133 & 235.08160 \\
\hline \multirow[t]{9}{*}{30} & 1 & -0.352853 & 0.368369 & -0.323835 & 0.340961 & 7.44037 & -0.319230 & 0.333509 & 0.288992 & 0.305176 & 9.28415 \\
\hline & 2 & -0.223356 & 0.235685 & -0.161288 & 0.178121 & 24.42412 & -0.190866 & 0.201625 & -0.127419 & 0.142794 & 41.19991 \\
\hline & 3 & -0.197719 & 0.208362 & -0.104892 & 0.124359 & 40.31589 & -0.165182 & 0.173360 & -0.070574 & 0.088725 & 95.39025 \\
\hline & 4 & -0.196240 & 0.205882 & -0.071025 & 0.093814 & 54.43312 & -0.162899 & 0.169841 & -0.038020 & 0.061304 & 177.04720 \\
\hline & 5 & -0.202003 & 0.210395 & -0.046135 & 0.075603 & 64.06616 & -0.172441 & 0.178293 & -0.014997 & 0.046725 & 281.57950 \\
\hline & 6 & -0.213804 & 0.221385 & -0.024700 & 0.063205 & 71.45019 & -0.185622 & 0.190458 & 0.002250 & 0.043550 & 337.33180 \\
\hline & 7 & -0.226688 & 0.233521 & -0.007941 & 0.057695 & 75.29344 & -0.200036 & 0.204048 & 0.018588 & 0.045106 & 352.37440 \\
\hline & 8 & -0.242599 & 0.248992 & 0.007775 & 0.057090 & 77.07155 & -0.217704 & 0.221309 & 0.033174 & 0.051831 & 326.98190 \\
\hline & 9 & -0.259471 & 0.265356 & 0.022036 & 0.060359 & 77.25358 & -0.235661 & 0.238850 & 0.046793 & 0.060639 & 293.88840 \\
\hline
\end{tabular}

Table 2 continued on next page 
AMER IBRAHIM AL-OMARI

\begin{tabular}{|c|c|c|c|c|c|c|c|c|c|c|}
\hline 10 & -0.276934 & 0.282548 & 0.036215 & 0.067383 & 76.15167 & -0.254437 & 0.257257 & 0.058627 & 0.069646 & 269.37800 \\
\hline 11 & -0.295302 & 0.300725 & 0.049094 & 0.074862 & 75.10616 & -0.273700 & 0.276336 & 0.072000 & 0.081003 & 241.14290 \\
\hline 12 & -0.313803 & 0.319255 & 0.062218 & 0.085295 & 73.28311 & -0.293398 & 0.295911 & 0.083363 & 0.091704 & 222.68060 \\
\hline 13 & -0.332279 & 0.337432 & 0.075374 & 0.095536 & 71.68733 & -0.311978 & 0.341101 & 0.095165 & 0.102770 & 231.90720 \\
\hline 14 & -0.351090 & 0.356205 & 0.087783 & 0.106535 & 70.09166 & -0.332096 & 0.334518 & 0.106272 & 0.113446 & 194.86980 \\
\hline 15 & -0.370555 & 0.375518 & 0.099545 & 0.116477 & 68.98231 & -0.352077 & 0.354327 & 0.118516 & 0.125081 & 183.27800 \\
\hline
\end{tabular}

Table 3. The Monte Carlo RMSEs and bias values of $H V_{m n}$ and $S H E_{m n}$ for the exponential distribution with $H[g(x)]=1$.

\begin{tabular}{|c|c|c|c|c|c|c|c|c|c|c|c|}
\hline \multirow[b]{3}{*}{$n$} & \multirow[b]{3}{*}{$m$} & \multicolumn{5}{|c|}{ SRS } & \multicolumn{5}{|c|}{ RSS } \\
\hline & & \multicolumn{2}{|c|}{$H V_{m n}$} & \multicolumn{2}{|c|}{$S H E_{m n}$} & \multirow[t]{2}{*}{$Q_{S H E_{m n}}$} & \multicolumn{2}{|c|}{$H V_{m n}$} & \multicolumn{2}{|c|}{$S H E_{m n}$} & \multirow[t]{2}{*}{$Q_{S H E_{m n}}$} \\
\hline & & Bias & RMSE & Bias & RMSE & & Bias & RMSE & Bias & RMSE & \\
\hline \multirow[t]{5}{*}{10} & 1 & -0.552032 & 0.677001 & -0.457584 & 0.600041 & 11.36778 & -0.430553 & 0.505229 & -0.342184 & 0.432785 & 14.33884 \\
\hline & 2 & -0.442683 & 0.571820 & -0.253108 & 0.442568 & 22.60362 & -0.337494 & 0.404667 & -0.148595 & 0.269907 & 33.30146 \\
\hline & 3 & -0.435444 & 0.561640 & -0.154607 & 0.391369 & 30.31675 & -0.332760 & 0.401125 & -0.052759 & 0.230412 & 42.55855 \\
\hline & 4 & -0.451545 & 0.575390 & -0.076188 & 0.371210 & 35.48550 & -0.348029 & 0.420617 & 0.025378 & 0.233566 & 44.47062 \\
\hline & 5 & -0.469437 & 0.597761 & 0.005489 & 0.372418 & 37.69784 & -0.366628 & 0.445977 & 0.101893 & 0.270512 & 39.34396 \\
\hline \multirow[t]{10}{*}{20} & 1 & -0.414064 & 0.490107 & -0.360711 & 0.445976 & 9.00436 & -0.357765 & 0.398661 & -0.312513 & 0.358752 & 10.01076 \\
\hline & 2 & -0.285717 & 0.376086 & -0.193143 & 0.310495 & 17.44043 & -0.234959 & 0.280262 & -0.140851 & 0.207405 & 25.99603 \\
\hline & 3 & -0.260773 & 0.351341 & -0.122104 & 0.272095 & 22.55530 & -0.213397 & 0.261261 & -0.072871 & 0.165700 & 36.57683 \\
\hline & 4 & -0.256116 & 0.352810 & -0.067569 & 0.251502 & 28.71461 & -0.210620 & 0.259248 & -0.017564 & 0.152350 & 41.23388 \\
\hline & 5 & -0.262412 & 0.358638 & -0.022414 & 0.244018 & 31.95980 & -0.214122 & 0.265246 & 0.022190 & 0.156584 & 40.96650 \\
\hline & 6 & -0.265650 & 0.360325 & 0.016823 & 0.248330 & 31.08166 & -0.218028 & 0.272315 & 0.061287 & 0.174543 & 35.90401 \\
\hline & 7 & -0.266934 & 0.365008 & 0.055461 & 0.256349 & 29.76894 & -0.224596 & 0.282196 & 0.103601 & 0.200858 & 28.82323 \\
\hline & 8 & -0.273952 & 0.377519 & 0.100674 & 0.274582 & 27.26671 & -0.232629 & 0.293062 & 0.145963 & 0.231970 & 20.84610 \\
\hline & 9 & -0.280123 & 0.381968 & 0.143573 & 0.293999 & 23.03046 & -0.236125 & 0.302083 & 0.188596 & 0.267430 & 11.47135 \\
\hline & 10 & -0.285183 & 0.391290 & 0.179545 & 0.322338 & 17.62171 & -0.238413 & 0.310922 & 0.231203 & 0.303760 & 2.30347 \\
\hline 30 & 1 & -0.367058 & 0.423423 & -0.332016 & 0.394742 & 6.77360 & -0.332526 & 0.361491 & -0.303272 & 0.334033 & 7.59576 \\
\hline
\end{tabular}

Table 3 continued on next page 


\section{NEW ENTROPY ESTIMATORS}

\begin{tabular}{rrrrrr|rrrrr}
2 & -0.233677 & 0.306086 & -0.173511 & 0.262016 & 14.39791 & -0.203455 & 0.236001 & -0.137679 & 0.182964 & 22.47321 \\
3 & -0.202277 & 0.281503 & -0.108684 & 0.223191 & 20.71452 & -0.170859 & 0.207468 & -0.078000 & 0.141567 & 31.76442 \\
4 & -0.194424 & 0.275072 & -0.067472 & 0.207505 & 24.56339 & -0.160246 & 0.199410 & -0.036059 & 0.123278 & 38.17863 \\
5 & -0.191705 & 0.272356 & -0.033792 & 0.197718 & 27.40457 & -0.159714 & 0.200465 & -0.002510 & 0.122595 & 38.84469 \\
6 & -0.186870 & 0.272196 & 0.000772 & 0.195841 & 28.05148 & -0.158702 & 0.202869 & 0.027994 & 0.128086 & 36.86270 \\
7 & -0.191094 & 0.275374 & 0.029066 & 0.198154 & 28.04186 & -0.161705 & 0.206226 & 0.059517 & 0.141042 & 31.60804 \\
8 & -0.195662 & 0.280589 & 0.056849 & 0.208607 & 25.65389 & -0.164468 & 0.212265 & 0.085540 & 0.160732 & 24.27767 \\
9 & -0.196983 & 0.282040 & 0.088082 & 0.220610 & 21.78060 & -0.165511 & 0.217222 & 0.115128 & 0.182796 & 15.84830 \\
10 & -0.197171 & 0.283394 & 0.115949 & 0.235447 & 16.91885 & -0.167152 & 0.220237 & 0.144441 & 0.205632 & 6.63149 \\
11 & -0.198853 & 0.286241 & 0.142656 & 0.253233 & 11.53154 & -0.173076 & 0.229318 & 0.172966 & 0.220033 & 4.04896 \\
12 & -0.204089 & 0.293653 & 0.171742 & 0.274080 & 6.66535 & -0.171555 & 0.232740 & 0.200259 & 0.214615 & 7.78766 \\
13 & -0.202908 & 0.298108 & 0.204980 & 0.228389 & 23.38717 & -0.176996 & 0.240454 & 0.231487 & 0.232102 & 3.47343 \\
14 & -0.205700 & 0.300842 & 0.232277 & 0.290007 & 3.60156 & -0.176922 & 0.244541 & 0.262425 & 0.211142 & 13.65780 \\
15 & -0.210699 & 0.305809 & 0.258234 & 0.300011 & 1.89595 & -0.177959 & 0.248760 & 0.291253 & 0.239115 & 3.87723 \\
\hline
\end{tabular}

Table 4. The Monte Carlo RMSEs and bias values of $H V_{m n}$ and $S H E_{m n}$ for the standard normal distribution and $H[g(x)]=1.419$.

\begin{tabular}{|c|c|c|c|c|c|c|c|c|c|c|c|}
\hline \multirow[b]{3}{*}{$n$} & \multirow[b]{3}{*}{$m$} & \multicolumn{5}{|c|}{ SRS } & \multicolumn{5}{|c|}{ RSS } \\
\hline & & \multicolumn{2}{|c|}{$H V_{m n}$} & \multicolumn{2}{|c|}{$S H E_{m n}$} & \multirow[t]{2}{*}{$Q_{S H E_{m n}}$} & \multicolumn{2}{|c|}{$H V_{m n}$} & \multicolumn{2}{|c|}{$S H E_{m n}$} & \multirow[t]{2}{*}{$Q_{S H E_{m n}}$} \\
\hline & & Bias & RMSE & Bias & RMSE & & Bias & RMSE & Bias & RMSE & \\
\hline \multirow[t]{5}{*}{10} & 1 & -0.598925 & 0.676499 & -0.499469 & 0.434171 & 35.8208955 & -0.484489 & 0.549750 & -0.388446 & 0.466743 & 15.09905 \\
\hline & 2 & -0.521455 & 0.591007 & -0.335907 & 0.436633 & 26.1205028 & -0.422169 & 0.471157 & -0.238609 & 0.320258 & 32.02733 \\
\hline & 3 & -0.563002 & 0.623188 & -0.275063 & 0.382983 & 38.5445484 & -0.462240 & 0.504378 & -0.181597 & 0.269765 & 46.51531 \\
\hline & 4 & -0.610651 & 0.663364 & -0.236072 & 0.351842 & 46.9609445 & -0.523019 & 0.557792 & -0.149270 & 0.244690 & 56.13239 \\
\hline & 5 & -0.671777 & 0.719069 & -0.200702 & 0.325688 & 54.7069892 & -0.584483 & 0.614209 & -0.111978 & 0.218489 & 64.42758 \\
\hline \multirow[t]{3}{*}{20} & 1 & -0.435480 & 0.483459 & -0.380981 & 0.434171 & 10.1948666 & -0.382986 & 0.420310 & -0.335512 & 0.377639 & 10.15227 \\
\hline & 2 & -0.327145 & 0.375798 & -0.231087 & 0.296133 & 21.1988888 & -0.275716 & 0.313472 & -0.182040 & 0.234712 & 25.12505 \\
\hline & 3 & -0.317948 & 0.364927 & -0.175301 & 0.251511 & 31.0790925 & -0.268657 & 0.304811 & -0.125104 & 0.189103 & 37.96057 \\
\hline
\end{tabular}

Table 4 continued on next page 


\section{AMER IBRAHIM AL-OMARI}

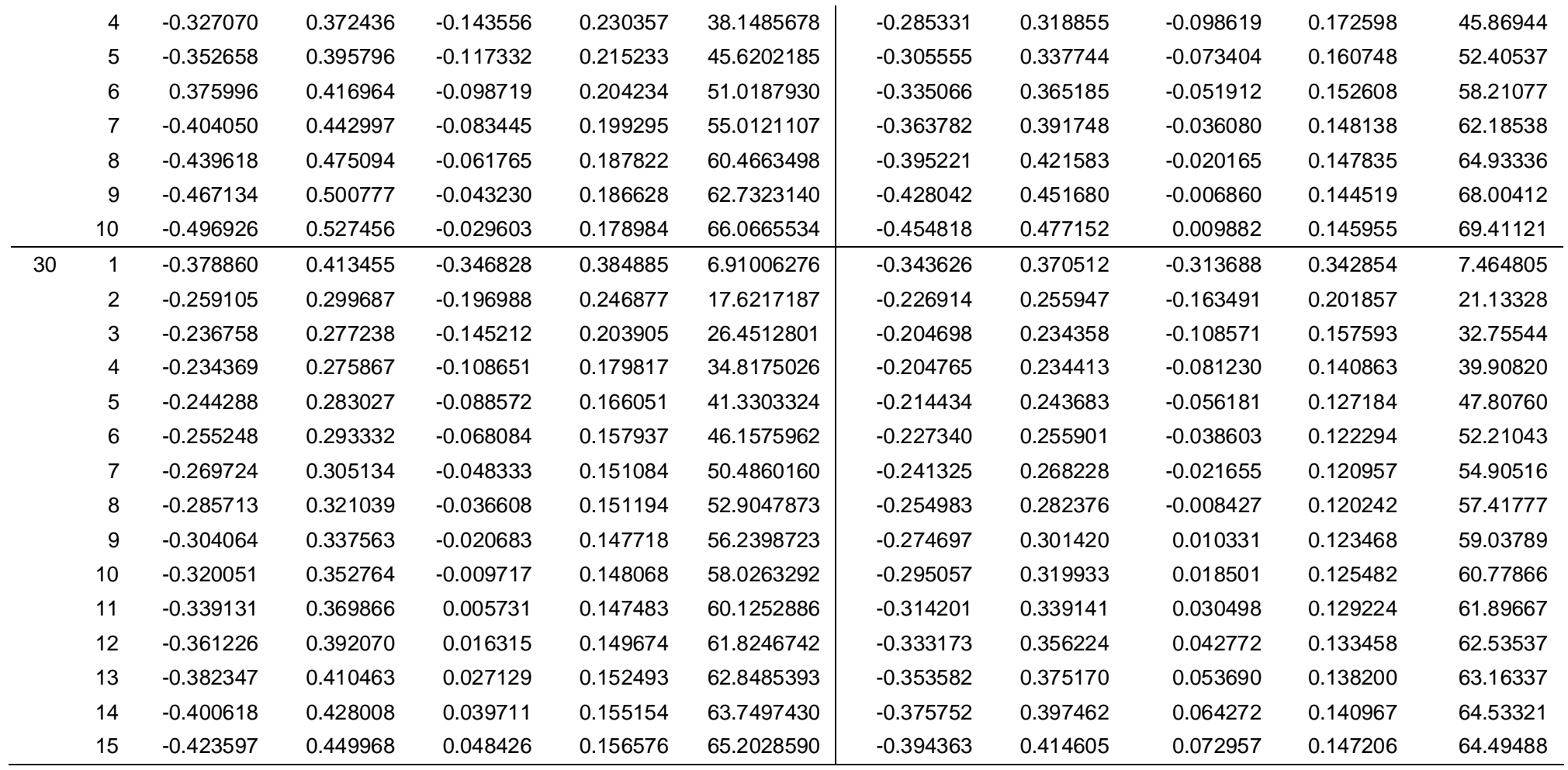

Table 5. The Monte Carlo RMSEs and bias values of $H V_{m n}$ and $S H E_{m n}$ for the uniform distribution with $H[g(x)]=0$ and exponential distribution with $H[g(x)]=1$ using DRSS.

SRS

RSS

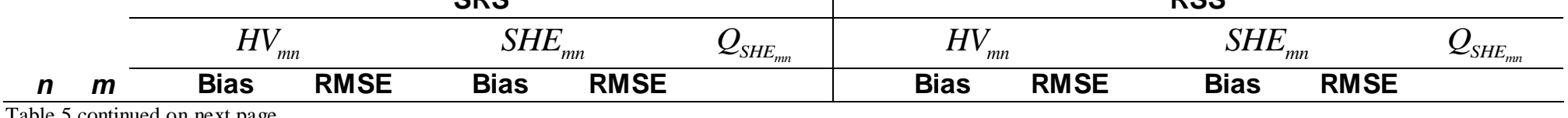




\section{NEW ENTROPY ESTIMATORS}

\begin{tabular}{|c|c|c|c|c|c|c|c|c|c|c|c|}
\hline \multirow[t]{5}{*}{10} & 1 & -0.327408 & 0.369593 & -0.230787 & 0.285326 & 22.7999448 & -0.365854 & 0.425279 & -0.267318 & 0.345821 & 18.68373 \\
\hline & 2 & -0.260621 & 0.278731 & -0.071592 & 0.121826 & 56.2926262 & -0.288898 & 0.340618 & -0.101687 & 0.207273 & 39.14796 \\
\hline & 3 & -0.296104 & 0.306116 & -0.014117 & 0.078474 & 74.3646199 & -0.300393 & 0.351750 & -0.018027 & 0.181245 & 48.47335 \\
\hline & 4 & -0.346305 & 0.352712 & 0.029482 & 0.073990 & 79.0225453 & -0.322839 & 0.377437 & 0.056521 & 0.201436 & 46.63056 \\
\hline & 5 & -0.404121 & 0.409902 & 0.065862 & 0.095121 & 76.7942093 & -0.335248 & 0.399189 & 0.134718 & 0.252269 & 36.80462 \\
\hline \multirow[t]{10}{*}{20} & 1 & -0.308453 & 0.329353 & -0.260588 & 0.285042 & 13.4539537 & -0.329105 & 0.363241 & -0.278366 & 0.317530 & 12.58421 \\
\hline & 2 & -0.189231 & 0.202666 & -0.095093 & 0.119561 & 41.0058915 & -0.204908 & 0.240316 & -0.112945 & 0.168444 & 29.90729 \\
\hline & 3 & -0.182095 & 0.191163 & -0.041993 & 0.071976 & 62.3483624 & -0.191216 & 0.228320 & -0.050530 & 0.133863 & 41.37044 \\
\hline & 4 & -0.197693 & 0.204342 & -0.010391 & 0.052373 & 74.3699288 & -0.190904 & 0.229986 & -0.003685 & 0.126728 & 44.89752 \\
\hline & 5 & -0.220876 & 0.225845 & 0.012711 & 0.049477 & 78.0924971 & -0.197900 & 0.239789 & 0.036502 & 0.139896 & 41.65871 \\
\hline & 6 & -0.247733 & 0.251580 & 0.035133 & 0.056178 & 77.6699261 & -0.207032 & 0.251002 & 0.078413 & 0.161731 & 35.56585 \\
\hline & 7 & -0.275808 & 0.278919 & 0.053697 & 0.068101 & 75.5839509 & -0.209883 & 0.258152 & 0.118656 & 0.192217 & 25.54115 \\
\hline & 8 & -0.303823 & 0.306608 & 0.071232 & 0.082285 & 73.1628007 & -0.218701 & 0.271560 & 0.158069 & 0.224230 & 17.42893 \\
\hline & 9 & -0.333903 & 0.336495 & 0.089491 & 0.098489 & 70.7309172 & -0.223692 & 0.278728 & 0.200103 & 0.262984 & 5.648518 \\
\hline & 10 & -0.363272 & 0.365731 & 0.106408 & 0.114566 & 68.6747910 & -0.228126 & 0.290431 & 0.244783 & 0.283888 & 2.252859 \\
\hline \multirow[t]{15}{*}{30} & 1 & -0.298092 & 0.312767 & -0.267592 & 0.283216 & 9.44824742 & -0.308011 & 0.331033 & -0.278838 & 0.304383 & 8.050557 \\
\hline & 2 & -0.170745 & 0.180210 & -0.107748 & 0.122162 & 32.2113090 & -0.182416 & 0.207785 & -0.118447 & 0.154790 & 25.50473 \\
\hline & 3 & -0.146113 & 0.153646 & -0.052193 & 0.070391 & 54.1862463 & -0.152039 & 0.180708 & -0.059074 & 0.114805 & 36.46933 \\
\hline & 4 & -0.149143 & 0.154886 & -0.023125 & 0.047458 & 69.3593998 & -0.145325 & 0.176699 & -0.019990 & 0.102139 & 42.19605 \\
\hline & 5 & -0.159888 & 0.164564 & -0.003052 & 0.038571 & 76.5617024 & -0.146632 & 0.179028 & 0.009230 & 0.105307 & 41.17847 \\
\hline & 6 & -0.174419 & 0.178204 & 0.013102 & 0.038421 & 78.4398779 & -0.149443 & 0.184598 & 0.038407 & 0.115953 & 37.18621 \\
\hline & 7 & -0.191854 & 0.194940 & 0.027534 & 0.046606 & 76.0921309 & -0.150245 & 0.188158 & 0.068588 & 0.133307 & 29.15156 \\
\hline & 8 & -0.209886 & 0.212509 & 0.040817 & 0.052754 & 75.1756396 & -0.153441 & 0.194332 & 0.095598 & 0.152215 & 21.67270 \\
\hline & 9 & -0.229010 & 0.231261 & 0.052824 & 0.061955 & 73.2099230 & -0.157250 & 0.199936 & 0.123844 & 0.175122 & 12.41097 \\
\hline & 10 & -0.248006 & 0.249993 & 0.065446 & 0.072283 & 71.0859904 & -0.162854 & 0.208891 & 0.151295 & 0.198703 & 4.877185 \\
\hline & 11 & -0.267506 & 0.269188 & 0.077163 & 0.082922 & 69.1955065 & -0.163540 & 0.213175 & 0.182129 & 0.207543 & 2.641961 \\
\hline & 12 & -0.287408 & 0.289018 & 0.088169 & 0.093391 & 67.6867877 & -0.167660 & 0.221482 & 0.207757 & 0.202062 & 8.768207 \\
\hline & 13 & -0.307160 & 0.308699 & 0.100118 & 0.104801 & 66.0507485 & -0.171024 & 0.225764 & 0.239466 & 0.211883 & 6.148456 \\
\hline & 14 & -0.327370 & 0.328890 & 0.111085 & 0.115458 & 64.8946456 & -0.170880 & 0.232977 & 0.268159 & 0.210502 & 9.646875 \\
\hline & 15 & -0.346997 & 0.348439 & 0.122960 & 0.126985 & 63.5560313 & -0.169873 & 0.235173 & 0.299068 & 0.210721 & 10.397450 \\
\hline
\end{tabular}




\section{AMER IBRAHIM AL-OMARI}

Table 6. The Monte Carlo RMSEs and bias values of $H V_{m n}$ and $S H E_{m n}$ for the standard normal distribution and $H[g(x)]=1.419$.

\begin{tabular}{|c|c|c|c|c|c|c|}
\hline \multirow[b]{2}{*}{$\mathbf{n}$} & \multicolumn{3}{|c|}{$H V_{m n}$} & \multicolumn{2}{|c|}{$S H E_{m n}$} & \multirow[t]{2}{*}{$Q_{S H E_{m n}}$} \\
\hline & m & Bias & RMSE & Bias & RMSE & \\
\hline \multirow[t]{5}{*}{10} & 1 & -0.415021 & 0.472162 & -0.316672 & 0.385139 & 18.43075 \\
\hline & 2 & -0.373395 & 0.412666 & -0.186378 & 0.256423 & 37.86185 \\
\hline & 3 & -0.427401 & 0.459119 & -0.143329 & 0.218981 & 52.30409 \\
\hline & 4 & -0.492911 & 0.518275 & -0.115918 & 0.202153 & 60.99503 \\
\hline & 5 & -0.554351 & 0.577281 & -0.084253 & 0.181100 & 68.62880 \\
\hline \multirow[t]{10}{*}{20} & 1 & -0.350703 & 0.383160 & -0.303014 & 0.340790 & 11.05804 \\
\hline & 2 & -0.245907 & 0.277809 & -0.152363 & 0.200155 & 27.95230 \\
\hline & 3 & -0.246496 & 0.276941 & -0.104439 & 0.162172 & 41.44168 \\
\hline & 4 & -0.262789 & 0.290545 & -0.078826 & 0.147712 & 49.16037 \\
\hline & 5 & -0.291340 & 0.317967 & -0.055774 & 0.138687 & 56.38321 \\
\hline & 6 & -0.316105 & 0.341597 & -0.037661 & 0.134214 & 60.70984 \\
\hline & 7 & -0.349246 & 0.373132 & -0.021199 & 0.132559 & 64.47397 \\
\hline & 8 & -0.384526 & 0.406764 & -0.008681 & 0.134158 & 67.01822 \\
\hline & 9 & -0.416151 & 0.436696 & 0.006082 & 0.132054 & 69.76066 \\
\hline & 10 & -0.445901 & 0.465518 & 0.023744 & 0.134764 & 71.05074 \\
\hline \multirow[t]{15}{*}{30} & 1 & -0.321940 & 0.345223 & -0.292331 & 0.318084 & 7.861300 \\
\hline & 2 & -0.206709 & 0.231560 & -0.143028 & 0.177006 & 23.55934 \\
\hline & 3 & -0.187163 & 0.212774 & -0.094482 & 0.138090 & 35.10015 \\
\hline & 4 & -0.190073 & 0.215577 & -0.066854 & 0.122350 & 43.24534 \\
\hline & 5 & -0.199843 & 0.224569 & -0.044224 & 0.111818 & 50.20773 \\
\hline & 6 & -0.214636 & 0.239021 & -0.025579 & 0.108667 & 54.53663 \\
\hline & 7 & -0.231613 & 0.255278 & -0.012061 & 0.108224 & 57.60543 \\
\hline & 8 & -0.247340 & 0.271084 & 0.001734 & 0.109348 & 59.66269 \\
\hline & 9 & -0.268298 & 0.291044 & 0.014961 & 0.113895 & 60.86674 \\
\hline & 10 & -0.286538 & 0.308661 & 0.027278 & 0.118811 & 61.50761 \\
\hline & 11 & -0.305310 & 0.326485 & 0.040250 & 0.123778 & 62.08769 \\
\hline & 12 & -0.324892 & 0.346062 & 0.051274 & 0.129747 & 62.50759 \\
\hline & 13 & -0.343097 & 0.363236 & 0.061548 & 0.135452 & 62.70964 \\
\hline & 14 & -0.369990 & 0.388586 & 0.070900 & 0.140756 & 63.77739 \\
\hline & 15 & -0.387740 & 0.406081 & 0.080947 & 0.145418 & 64.18990 \\
\hline
\end{tabular}




\section{NEW ENTROPY ESTIMATORS}

Table 7. The Monte Carlo RMSEs and bias values of $A H E j_{m n}, j=$ SRS, RSS, DRSS (AI-Omari, 2014).

\begin{tabular}{|c|c|c|c|c|c|c|c|c|c|c|}
\hline \multirow[b]{2}{*}{$n$} & \multirow[b]{2}{*}{$m$} & \multicolumn{2}{|c|}{$\operatorname{AHESRS}_{m n}$} & \multirow[t]{2}{*}{$Q_{A H E S R S}$} & \multicolumn{2}{|c|}{$\operatorname{AHERSS}_{m n}$} & \multirow[t]{2}{*}{$Q_{A H E R S S}$} & \multicolumn{2}{|c|}{ AHEDRSS $_{m n}$} & \multirow[t]{2}{*}{$Q_{\text {AHEDRSS }}$} \\
\hline & & Bias & RMSE & & Bias & RMSE & & Bias & RMSE & \\
\hline \multicolumn{11}{|c|}{ Uniform distribution with $H[g(x)]=0$} \\
\hline \multirow[t]{2}{*}{10} & 2 & -0.298609 & 0.350332 & 22.554260 & -0.189664 & 0.228762 & 30.516686 & -0.145388 & 0.176159 & 36.799638 \\
\hline & 3 & -0.249056 & 0.298944 & 34.126897 & -0.154894 & 0.186380 & 45.818350 & -0.122180 & 0.144286 & 52.865580 \\
\hline \multirow[t]{2}{*}{20} & 4 & -0.144016 & 0.167779 & 38.917933 & -0.100304 & 0.118284 & 46.844385 & -0.082268 & 0.096978 & 52.541328 \\
\hline & 5 & -0.133179 & 0.157805 & 45.393360 & -0.091608 & 0.108584 & 55.163743 & -0.077708 & 0.091093 & 59.665700 \\
\hline \multirow[t]{2}{*}{30} & 7 & -0.092957 & 0.109089 & 53.285144 & -0.066053 & 0.077716 & 61.912883 & -0.058041 & 0.067650 & 65.297015 \\
\hline & 8 & -0.089259 & 0.105818 & 57.501446 & -0.064713 & 0.076188 & 65.573926 & -0.056421 & 0.065369 & 69.239420 \\
\hline \multicolumn{11}{|c|}{ Exponential distribution with $H[g(x)]=1$} \\
\hline \multirow[t]{2}{*}{10} & 2 & -0.323532 & 0.483573 & 15.432654 & -0.220406 & 0.315220 & 22.103853 & -0.173991 & 0.251460 & 26.175364 \\
\hline & 3 & -0.265713 & 0.443276 & 21.074710 & -0.159787 & 0.276197 & 31.144406 & -0.128545 & 0.223802 & 36.374698 \\
\hline \multirow[t]{2}{*}{20} & 4 & 0.141143 & 0.279706 & 20.720501 & -0.098056 & 0.179990 & 30.572271 & -0.075338 & 0.179771 & 21.833938 \\
\hline & 5 & 0.118697 & 0.271887 & 24.189015 & -0.072456 & 0.172661 & 34.905333 & -0.052175 & 0.145269 & 39.417988 \\
\hline \multirow[t]{2}{*}{30} & 7 & -0.058550 & 0.205261 & 25.461009 & -0.027194 & 0.130283 & 36.825134 & -0.046556 & 0.115023 & 38.868929 \\
\hline & 8 & -0.036080 & 0.200329 & 28.604115 & -0.010631 & 0.136358 & 35.760488 & -0.001239 & 0.120306 & 38.092543 \\
\hline \multicolumn{11}{|c|}{ Standard normal distribution with $H[g(x)]=1.419$} \\
\hline \multirow[t]{2}{*}{10} & 2 & -0.409842 & 0.496627 & 15.969354 & -0.308706 & 0.375690 & 20.262250 & -0.262149 & 0.316029 & 23.417728 \\
\hline & 3 & -0.386562 & 0.468471 & 24.826698 & -0.291133 & 0.353844 & 29.845470 & -0.254450 & 0.303820 & 33.825435 \\
\hline \multirow[t]{2}{*}{20} & 4 & -0.214227 & 0.279269 & 25.015573 & -0.168035 & 0.219922 & 31.027583 & -0.148107 & 0.194728 & 32.978368 \\
\hline & 5 & -0.205782 & 0.272804 & 31.074594 & -0.160392 & 0.213700 & 36.727225 & -0.145734 & 0.191755 & 39.693427 \\
\hline \multirow[t]{2}{*}{30} & 7 & -0.132038 & 0.196792 & 35.506368 & -0.105796 & 0.158654 & 40.851067 & -0.095517 & 0.143483 & 43.793433 \\
\hline & 8 & -0.129915 & 0.193509 & 39.724146 & -0.102504 & 0.157726 & 44.143270 & -0.094560 & 0.145579 & 46.297458 \\
\hline
\end{tabular}




\section{AMER IBRAHIM AL-OMARI}

\section{Conclusion}

Three entropy estimators are suggested using SRS, RSS, and DRSS methods. The consistency of these estimators is proved as well as some properties are reported. Based on theoretical and numerical comparisons the suggested entropy estimators are more efficient than Vasicek (1976) and Al-Omari (2014) entropy estimators. However, the suggested estimators of entropy in this paper can be extended by considering other sampling methods such as the multistage RSS and median RSS methods.

\section{Acknowledgements}

The author thanks the referees for their helpful and valuable comments that substantially improved this paper.

\section{References}

Al-Omari, A. I. (2014). Estimation of entropy using random sampling. Journal of Computation and Applied Mathematics, 261, 95-102. doi:10.1016/j.cam.2013.10.047

Al-Saleh, M. F. \& Al-Kadiri, M. A. (2000). Double ranked set sampling. Statistics and Probability Letters, 48(2), 205-212. doi:10.1016/S0167-7152(99)00206-0

Choi, B. (2008). Improvement of goodness of fit test for normal distribution based on entropy and power comparison. Journal of Statistical Computation and Simulation, 78(9), 781-788. doi:10.1080/00949650701299451

Choi, B., Kim, K., \& Song, S. H. (2004). Goodness of fit test for exponentiality based on Kullback-Leibler information. Communication in Statistics-Simulation and Computation, 33(2), 525-536.

doi:10.1081/SAC-120037250

Goria, M. N., Leonenko, N. N., Mergel, V. V., \& Novi Inverardi, P. L. (2005). A new class of random vector entropy estimators and its applications in testing statistical hypotheses. Journal of Nonparametric Statistics, 17(3), 277-297. doi:10.1080/104852504200026815 


\section{NEW ENTROPY ESTIMATORS}

Correa, J. C. (1995). A new estimator of entropy. Communication in Statistics-Theory Methods, 24(10), 2439-2449. doi:10.1080/03610929508831626

Ebrahimi, N., Pflughoeft, K., \& Soofi, E. S. (1994). Two measures of sample entropy. Statistics \& Probability Letters, 20(3), 225-234. doi:10.1016/0167-7152(94)90046-9

Mahdizadeh, M. (2012). On the use of ranked set samples in entropy based test of fit for the Laplace distribution. Revista Colombiana de Estadística, 35(3), 443-455.

McIntyre, G. A. (1952). A method for unbiased selective sampling using ranked sets. Australian Journal of Agricultural Research, 3(4), 385-390. doi:10.1071/AR9520385

Noughabi, H. A. \& Noughabi, R. A. (2013). On the entropy estimators. Journal of Statistical Computation and Simulation, 83(4), 784-792. doi:10.1080/00949655.2011.637039

Noughabi, H. A. \& Arghami, N. R. (2010). A new estimator of entropy. Journal of the Iranian Statistical Society, 9(1), 53-64.

Park, S. \& Park, D. (2003). Correcting moments for goodness of fit tests based on two entropy estimates. Journal of Statistical Computation and Simulation, 73(9), 685-694. doi:10.1080/0094965031000070367

Shannon, C. E. (1948a). A mathematical theory of communications. Bell System Technical Journal 27(3), 379-423. doi:10.1002/j.1538-7305.1948.tb01338.x

Shannon, C. E. (1948b). A mathematical theory of communications. Bell System Technical Journal 27(4), 623-656. doi:10.1002/j.1538-7305.1948.tb00917.x

Takahasi, K. \& Wakimoto, K. (1968). On the unbiased estimates of the population mean based on the sample stratified by means of ordering. Annals of the Institute of Statistical Mathematics, 20(1), 1-31. doi:10.1007/BF02911622

Theil, J. (1980). The entropy of maximum entropy distribution. Economics Letters, 5(2), 145-148. doi:10.1016/0165-1765(80)90089-0

Van Es, B. (1992). Estimating functionals related to a density by class of statistics based on spacings. Scandinavian Journal of Statistics, 19(1), 61-72.

Vasicek, O. (1976). A test for normality based on sample entropy. Journal of the Royal Statistical Society, B, 38, 54-59. 


\section{AMER IBRAHIM AL-OMARI}

Wieczorkowski, R. \& Grzegorzewsky, P. (1999). Entropy estimators improvements and comparisons. Communication in Statistics-Simulation and Computation, 28(2), 541-567. doi:10.1080/03610919908813564 